\title{
The Leucine Binding Proteins of Escherichia coli as Models for Studying the Relationships Between Protein Structure and Function
}

\author{
Tammy K. Antonucci, Robert Landick, and Dale L. Oxender \\ Department of Biological Chemistry, University of Michigan, Ann Arbor, Michigan 48109
}

The genes encoding the leucine binding proteins in $E$ coli have been cloned and their DNA sequences have been determined. One of the binding proteins (LIV$\mathrm{BP}$ ) binds leucine, isoleucine, valine, threonine, and alanine, whereas the other (LS-BP) binds only the D- and L-isomers of leucine. These proteins bind their solutes as they enter the periplasm, then interact with three membrane components, livH, livG, and livM, to achieve the translocation of the solute across the bacterial cell membrane. Another feature of the binding proteins is that they must be secreted into the periplasmic space where they carry out their function. The amino acid sequence of the two binding proteins is $80 \%$ homologous, indicating that they are the products of an ancestral gene duplication. Because of these characteristics of the leucine binding proteins, we are using them as models for studying the relationships between protein structure and function.

Key words: amino acid transport, binding proteins, secretion, gene duplication, oligonucleotidedirected mutagenesis

There are three major systems operating in Escherichia coli for the transport of the branched-chain amino acids. Two of these systems consist of a binding protein and three membrane components while the third system appears to involve only a single membrane component. In this discussion we will describe the biochemical and genetic properties of the two binding protein-dependent transport systems, emphasizing the multiple functions of the binding protein components of the transport systems and, as a consequence, the value of the binding proteins for understanding the relationship(s) between protein structure and function.

The LIV-I system transports L-leucine, L-isoleucine, and L-valine with $\mathrm{K}_{\mathrm{d}}$ values of approximately $10^{-6}$ to $10^{-7} \mu \mathrm{M}$ [1-4]. In addition, this system is responsible for the transport of threonine and alanine, but with somewhat lower affinities [2].

Robert Landick's present address is the Department of Biological Sciences, Stanford University, Stanford, CA 94305.

Received June 6, 1985; revised and accepted August 6, 1985.

(C) 1985 Alan R. Liss, Inc. 
The other transport system to be considered is the leucine-specific transport system (LS). The LS system transports L-leucine with a $\mathrm{K}_{\mathrm{d}}$ value of approximately $10^{-6}$ $\mu \mathrm{M}$; D-leucine is also an acceptable substrate but with a lower affinity [1-4]. All structural genes for the LS and LIV-I transport systems are located at the minute 76 region on the $E$ coli chromosome [4].

The genes encoding the components of the branched-chain amino acid transport systems LIV-I and LS in E coli have been cloned [5]. The gene organization is shown in Figure 1. There are three functional operons with their directions of transcription indicated by the arrows. The liv $J$ and livK gene products are the periplasmic binding proteins, called LIV-BP and LS-BP, respectively. The LIV-BP binds isoleucine, leucine, and valine with high affinity and threonine and alanine with a somewhat lower affinity. The LS-BP binds only isomers of leucine. The livH, livM, and livG genes encode proteins that have been localized to the inner membrane of the bacterial cell [6]. These proteins presumably interact with the BP-amino acid complex to effect the transport of the amino acid into the cytoplasm of the cell. Thus the LIV-I and LS transport systems consist of individual binding proteins that have different substrate specificities yet interact with the same membrane components during the transport process. The livL operon produces a $17 \mathrm{kD}$ protein but its role in transport, if any, has not been determined [7].

\section{CHARACTERISTICS OF THE LIV-BP AND THE LS-BP}

The DNA sequence of the livJ and livK genes encoding the two binding proteins has been determined [8] and is shown in Figure 2. A comparison of the coding regions of the two sequences shows that the two binding protein genes are $80 \%$ homologous, indicating that they are the products of an ancestral gene duplication [8-11]. While the sequences of the $5^{\prime}$ flanking regions and coding regions revealed no strong homology with the known ribosome-binding sites, the pattern of codon usage in both genes implies that they are translated with average efficiency.

The amino acid sequence of the LIV-BP has been previously reported [12] and is consistent with the DNA sequence except for two positions [8]. Moreover, the crystal structure of the mature LIV-BP was determined to a resolution of $2.0 \mathrm{~A}$ [13]. The crystal structure reveals that the LIV-BP has two domains that fold to form a cleft [13]. One domain of LIV-BP is composed of the N-terminal half of the protein, while the other domain is formed by most of the $\mathrm{C}$ terminal portion of the molecule. The secondary structure obtained from the crystallographic analysis shows that each domain is formed by an alternating series of alpha helices and beta sheets [8]. Figure 3 shows the predicted secondary structural features superimposed onto a drawing of the crystal structure. The filled-in dots represent differences in amino acid sequences between the two binding proteins. The two arrows in Figure 2 point out regions where amino acid insertions occur in the LS-BP. A disulfide bridge can be positioned between segments beta- 2 and beta- 3 .

\section{Substrate Binding}

One of the major functions carried out by the BPs is binding of substrate. As mentioned above, the LIV-BP and the LS-BP have different substrate specificities. Saper and Quiocho [13] have observed that when L-leucine is diffused into crystals 


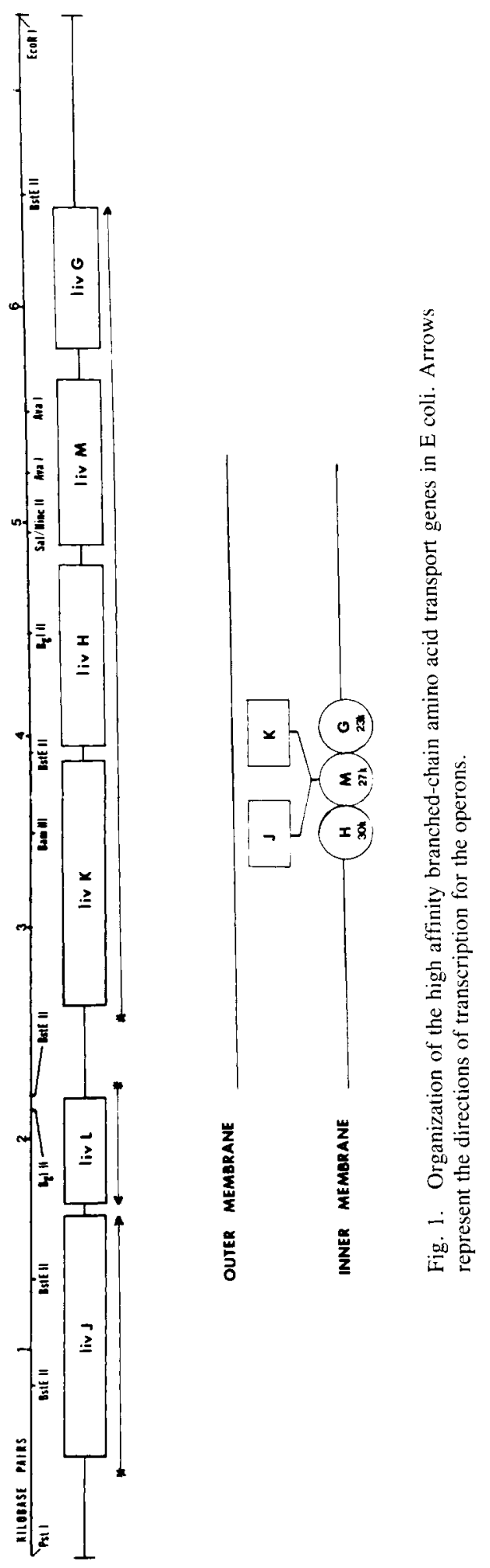




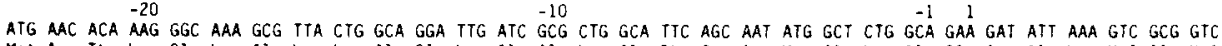

ATG AAC ACA 20 aAG GGC AAA GCG ITA CTG GCA GGA TTG ATC GCG CTG GCA TIC AGC AAT ATG GCT CTG GCA GAA GAT ATT AAA GTC GCG GTC

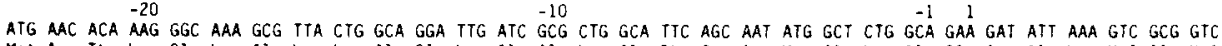
Met Asn Inr Lys Gly Lys Ala Leu Leu Ala Gly Leu lle Ala Leu Ala Phe Ser Asn Met Ala Leu Ala Glu Asp lle Lys val Ala Val Met Lys Arg Asn Ala Lys Thr lle Ile Ala Gly Met lle Ala Leu Ala lle Ser His thr Ala Met Ala Asp Asp lle Lys Val Ala Val ATG AAA CGG AAT GCG AAA ACT ATC ATC GCA GGG ATG ATT GCA CTG GCA ATT TCA CAC ACC GCT ATG GCT GAC GAT ATT AAA GTC GCC GIT GTG GGC GCA ATG TCC GGT CCG GII GCG CAG TAC GGT GAC CAG GAG III ACC GGC GCA GAG CAG GCG GIT GCG GAT ATC AAC GCT AAA GGC Val Gly Ala Met Ser Gly Pro Val Ala G!n Tyr Gly Asp Gin Glu Phe Thr Gly Ala Glu Gin Ala Val Ala Asp lle Asn Ala Lys Gly val Gly Ala Met Ser Gly Pro lle Ala Gln Tro Gly Ile Met Glu Phe Asn Gly Ala Glu Gin Ala lle Lys Asp lle Asn Ala Lys Gly
GTC GGC GCG ATG ICC GGC CCG ATT GCC CAG TGG GGC ATA ATG GAA ITT ARC GGC GCG GAG CAG GCG ATT AAA GAC ATT AAT GCC AAA GGG

GGC AIT AAA GGC AAC AAA CTG CAA ATC GCA AAA TAT GAC GAT GCC TGT GAT CCG AAA CAG GCG GTT GCG GTG GCG AAC AAA GTC GTT AAC

GGC AIT AAA GGC AAC AAA CTG CAA ATC GCA AAA TAT GAC GAT GCC TGT GAT CCG AAA CAG GCG GTT GCG GTG GCG AAC AAA GTC GTT AAC

GGC AIT AAA GGC AAC AAA CTG CAA ATC GCA AAA TAT GAC GAT GCC TGT GAT CCG AAA CAG GCG GTT GCG GTG GCG AAC AAA GTC GTT AAC Gly lle Lys Gly Asn Lys Leu Gin Ile Ala Lys Iyr Asp Asp Ala Cys Asp Pro Lys Gin Ala Val Ala Val Ala Asn Lys Val Val Asn Gly lle Lys Gly Asp Lys Leu Val Gly Val Glu Tyr Asp Asp Ala Cys Asp Pro Lys Gin Ala Val Ala Val Ala Asn Lys lle Val As GGA ATT AAG GGC GAT AAA CTG GTT GGC GIG GAA TAT GAC GAC GCA TGC GAC CCG AAA CAA GCC GTT GCG GIC GCC AAC AAM ATC GIT AAT GAC GGC AIt AAA IAT GTG ATT GGT CAC CIC TGI TCC TCA TCA ACG CAG CCT GCG TCG GAT ATC TAC GAA GAC GAA GGC ATT IIG ATG ATC GAC GGC AIT AAA IAT GTG ATT GGT CAC CIC TGI TCC TCA TCA ACG CAG CCT GCG TCG GAT ATC TAC GAA GAC GAA GGC AIT IIG ATG ATC Asp Gly Ile Lys Tyr Val lle Gly His Leu Cys Ser Ser Ser Thr Gln Pro Ala Ser Asp Ile Tyr Glu Asp Glu Gly lle Leu Met lle Asp Gly lle Lys Tyr Val lle Gly His Leu Cys Ser Ser Ser Thr GTn Pro Ala Ser Asp Mle Tyr Glu Asp Glu Gly Ile Leu Met Ile GAC GGC ATT AAA TAC GTT ATT GGT CAT CTG TGT TCT TCT TCT ACC CAG CCT GCG TCA GAC ATC TAT GAA GAC GAA GGT aTT CTA ATG ATC

100 110 ACC CCA GCG GCA ACC GCG CCG GAG CTG ACC GCC CGI GGC TAT CAG CTG ATC CTG CGA RCC ACC GGC CTG GAT TCC GAC CAA GGG CCG ACS Thr Pro Ala Ala Thr Ala Pro Glu Leu Thr Ala Arg Gly Tyr Gin Leu lle Leu Arg Th Thr Gly Leu Asp Ser Asp Gin Gly Pro Thr Ser Pro Gly Ala Thr Ala Pro Glu Leu Thr Gln Arg Gly Tyr Gln His lle Met Arg Thr Ala Gly Leu Asp Ser Ser Gln Gly Pro Thr ICG CCG GGA GCG ACC GCG CCG GAA CTA ACC CAA CGC GGT IAT CAA CAC ATT ATG CGT ACT GCC GGG CTG GAC TCT TCC CAG GGG CCA ACG

GCT GCC AAA TAT ATI CIT GAG AAA GTG AAA CCG CAG CGT ATT GCT ATC GTT CAC GAC AAA CAG CAA TAC GGC GAA GGT GTG GCG CGA GCG

GCT GCC AAA TAT ATI CIT GAG AAA GTG AAA CCG CAG CGT ATT GCT ATC GTT CAC GAC AAA CAG CAA TAC GGC GAA GGT CTG GCG CGA GCG

GCT GCC AAA TAT ATI CIT GAG AAA GTG AAA CCG CAG CGT ATT GCT ATC GTT CAC GAC AAA CAG CAA TAC GGC GAA GGT GTG GCG CGA GCG Ala Ala Lys Tyr Ile Leu Glu Lys Val Lys Pro Gin Arg lle Ala lle Val His Asp Lys Gin Gin Tyr Gly Glu Gly Leu Ala Arg Ala Ala Ala Lys Tyr Ile Leu Glu Thr Val Lys Pro Gin Arg lle Ala yle lle His Asp Lys Gln Gln Tyr Gly Glu Gly Leu Ala Arg Ser GCG GCA AAA TAC ATT CTT GAG ACG GTG AAG CCC CAG CGC ATC GCC ATC ATC CAC GAC AAA CAA CAG IAT GGC GAA GGG CTG GCG CGT ICG GTG CAG GAC GGC CTG AAG AAA GGC AAT GCA AAC GTG GTG TIC ITT GAI GGC ATC ACC GCC GGG GAA AAA GAT IIC TCA ACG CTG GTG GCG GTG CAG GAC GGC CTG AAG AAA GGC AAT GCA AAC GTG GTG ITC ITT GAI GGC ATC ACC GCC GGG GAA AAA GAT IIC TCA ACG CTG GTG GCG GTG CAG GAC GGC CTG AAG AAA GGC AAT GCA AAC GTG GTG TIC ITT GAI GGC ATC ACC GCC GGG GAA AAA GAT IIC TCA ACG CTG GTG GCG Val Gin Asp Gly Leu Lys Lys Giy Asn Ala Asn Val Val Phe Phe Asp Giy lle Thr Ala Gly Glu Lys Asp Phe Ser Thr Leu Val Ald Val Gln Asp Gly Leu Lys Ala Ala Asn Ala Asn Val Val Phe Phe Asp Gly lle Thr Ala Gly Glu Lys Asp Phe Ser Ala Leu Ile Ala GIG CAG GAC GGG CTG AAA GCG GCI AAC GCC AAC GTC GTC TTC IIC GAT GGT ATT ACC GCC GGG GAG AAA GAT TTC TCC GCG CTG ATC GCC

190 200 210 CGT CTG AaA AAA GAG aAT ATC GAC TTC GIT TAC TAC GGC GGT TAT CAC CCG GAA ATG GGG CAA ATC CTG CGT CAG GCA CGC GCG GCA GGG Arg Leu Lys Lys Glu Asn lle Asp Phe Val Tyr Tyr Gly Gly Tyr His Pro Glu Met Gly Gin tle Leu Arg Gin Ala Arg Ala Ala Gly Arg Leu Lys Lys Giu Asn Ile Asp Phe Val Tyr Tyr Giy Gly Tyr Tyr Pro Glu Met Gly Gin Met Leu Arg Gin Ala Arg Ser Val Giy CGC CTG AAA AAA GAA AAC ATC GAC TIC GTT TAC TAC GGC GGT TAC IAC CCG GAA ATG GGG CAG ATG CTG CGC CAG GCC CGT Ter GIT GGC CTG AAA ACT CAG ITT ATG GGg CCG GAA GGI GTG GCT AAC GTT TCG CTG TCT AAC ATT GCG GGC GAA TCA GCG GAA GGG CTA CTG GTG ACC CTG AaA ACT CAG IIT ATG GGG CCG GAA GGI GTG GCT AAC GTT TCG CTG ICT AAC ATT GCG GGC GAA 240 TCA GCG GAA GGG CTA CTG GTG ACC 240 Leu Lys Thr Gln Phe Met Gly Pro Glu Gly Val Ala Asn Val Ser Leu Ser Asn Ile Ala Gly Giu Ser Ala Giu Gly Leu Leu Val Thr Leu Lys Thr GIn Phe Met Giy Pro Glu Gly Val Giy Asri Ala Ser Leu Ser Asn Ile Ala Gly Asp Ala Ala Giu Gly Met Leu Val Thr Leu Lys inr GIn Phe Met Giy Pro Glu Giy Val Giy Asri Ala Ser Leu Ser Asn Ile Ala Giy Asp Aid Ald Giu Gly Met Leu val Thr

AAA CCG 250 AAG AAC TAC GAT CAG GTT CCG GCG AAC AAA CCC ATT GTT GAC GCG ATC AAA GCG AAA AAA CAG GAC CCA AGT GGC GCA TTC GTT

AAA CCG 250 AAG AAC TAC GAT CAG GTT CCG GCG AAC AAA CCC ATT GTT GAC GCG ATC AAA GCG AAA AAA CAG GAC CCA AGT GGC GCA TTC GTT

AAA CCG 250 AAG AAC TAC GAT CAG GTT CCG GCG AAC AAA CCC ATT GTT GAC GCG ATC AAA GCG AAA AAA CAG GAC CCA AGT GGC GCA TTC GTT AAA CCG AAG AAC JAC GAT CAG GTT CCG GCG AAC AAA CCC ATT GTT GAC GCG ATC AAA GCG AAA AAA CAG GAC CCA AGT GGC GCA TTC GTT
Lys Pro Lys Asn Tyr Asp Gln Val Pro Ala Asn Lys Pro lle Val Asp Ala Ile Lys Ala lys Lys Gin Asp Pro Ser Gly Ala Phe Val Met Pro Lys Arg Tyr Asp Gln Asp Pro Ala Asn Gin Gly lle Val Asp Ala Leu Lys Ala Asp Lys Lys Asp Pro Ser Gly Pro Tyr Val ATG CCA AAA CGC TAT GAC CAG GAT CCG GCA AAC CAG GGC ATC GTT GAT GCG CTG AAA GCA GAC AAG AAA GAT CCG TCC GGG CCT TAT GTC

280 TGG ACC ACC TAC GCC GCG CTG CAA TCT TTG CAG GCG GGC CTC AAT CAG Trp Thr Thr Tyr Ala Ala Leu Gin Ser Leu Gin Ala Gly Leu Asn Gin Irp lle Thr Tyr Ala Ala Val Gin Ser Leu Ala Thr Ala Leu Glu Arg Thr Gly Ser Asp Glu Pro Leu Ala Leu Val Lys Asp Leu Lys
IGG ATC ACC TAC GCG GCG GIG CAA TCT CTG GCG ACT GCC CIT GAG CGT ACC GGC AGC GAT GAG CCG CTG GCG CTG GTG AAA GAT ITA AAA 310 320 GCG AAC ICC GTG GAT ACC GTA ATG GGC CCG CTG ACC TGG GAT GAG AAA GGC GAT CTG AAA GGC ITT GAG TTC GGC GTA TTT GAC TGG CAC Ala Asn Ser Val Asp Thr Val Met Gly Pro Leu Thr Trp Asp Glu Lys Gly Asp Leu Lys Gly Phe Glu Phe Gly Val Phe Asp Irp His Ala Asn Gly Ala Asn Thr Val Ile Gly Pro Leu Asn Trp Asp Glu Lys Gly Asp Leu Lys Gly Phe Asp Phe Gly Val Phe Gln Trp his GCT AAC GGT GCA AAC ACC GTG ATT GGG CCG CTG AAC TGG GAT GAA AAA GGC GAT CTT AAG GGA TTT GAT TTT GGT GTG TTC CAG TGG CAC GCC AAC GGC ACG GCC ACC GAT GCG 344 Ald Asn Gly Thr Ald Thr Asp Ala Lys Ala Asp Gly Ser Ser Thr Ala Ala Lys LIV-BP LS $-\mathrm{BP}$

Fig. 2. Nucleotide sequences of the genes encoding LIV-BP and LS-BP. Positive numbering indicates the mature protein sequences, while negative numbering designates the signal sequences. Homologous amino acids are noted by asterisks. 


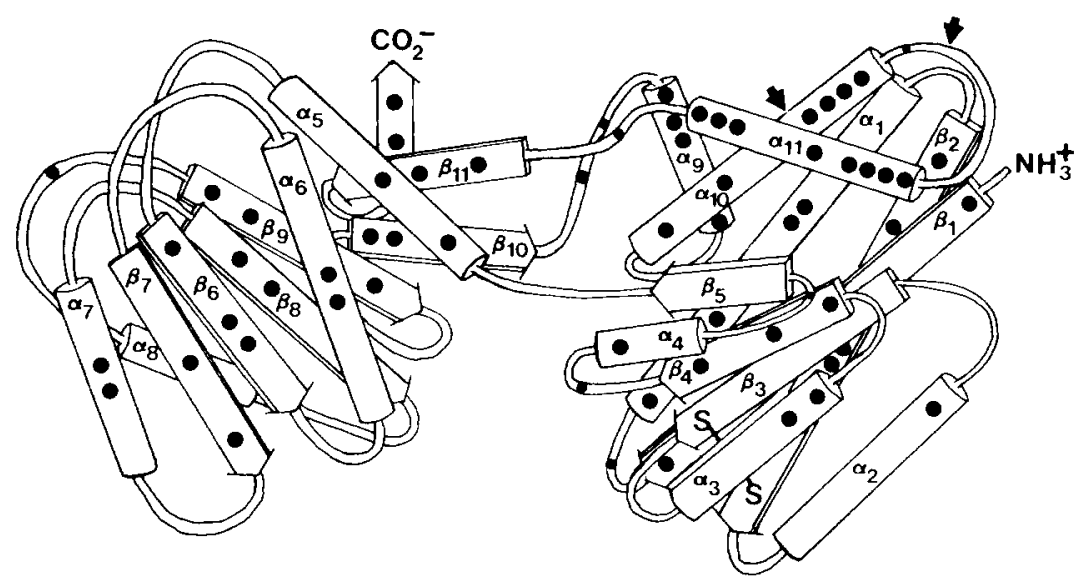

Fig. 3. Structure of the LIV-BP. Predicted secondary structures are superimposed on the threedimensional structure reported by Saper and Quiocho [13]. Filled circles indicate the positions of amino acid differences between the LIV-BP and LS-BP sequences. The two dark arrows indicate the region where a two-amino acid insertion occurs in the LS-BP sequence. A disulfide bridge is indicated between segments beta-2 and beta- 3 .

of the LIV-BP, it binds to the inside face of the N-terminal domain. It is reasonable to suggest [8] that amino acid differences between the two BP sequences within the cleft between the two domains are responsible for the different substrate specificities of the LIV-BP and LS-BP. Nonhomologous residues are present near the cleft in segments beta-1-alpha-1, beta-4-alpha-4, alpha-9, alpha-10, beta-9, and beta-10. Thus, these areas of the BPs may be involved either in forming the structure of the substrate binding site or in directly interacting with the substrate.

\section{INTERACTION OF BPS WITH MEMBRANE COMPONENTS}

Both the LIV-BP-substrate complex and the LS-BP-substrate complex must interact with the same three membrane components to effect the transport of the substrate. Therefore, it is interesting to note that some of the regions of high amino acid sequence conservation include the $\mathrm{C}$ terminus of beta-2, alpha- 2 , beta-3, alpha- 3 , the $\mathrm{C}$ terminus of beta- 8 , and alpha- 8 . Because of their sequence conservation and position on the BPs, these regions may function in interactions with the membrane components. It is tempting to speculate that this interaction may be the signal that initiates the membrane translocation process.

\section{SECRETION OF THE BPS INTO THE PERIPLASM}

Both the LIV-BP and LS-BP are synthesized as precursors with a 23-amino acid signal sequence that is processed in association with the BP secretion into the periplasm [7, 10, 14]. Using the rules of Chou and Fasman [15], the secondary structure of the N-terminal portion of the precursor would be predicted to form a helical hairpin structure. These two helices would form from the signal sequence and the mature $\mathrm{N}$-terminal regions and would contain hydrophobic amino acids in the sections that might span the membrane. A model for the secretion of the BPs is shown in Figure 
214:JCB Antonucci, Landick, and Oxender

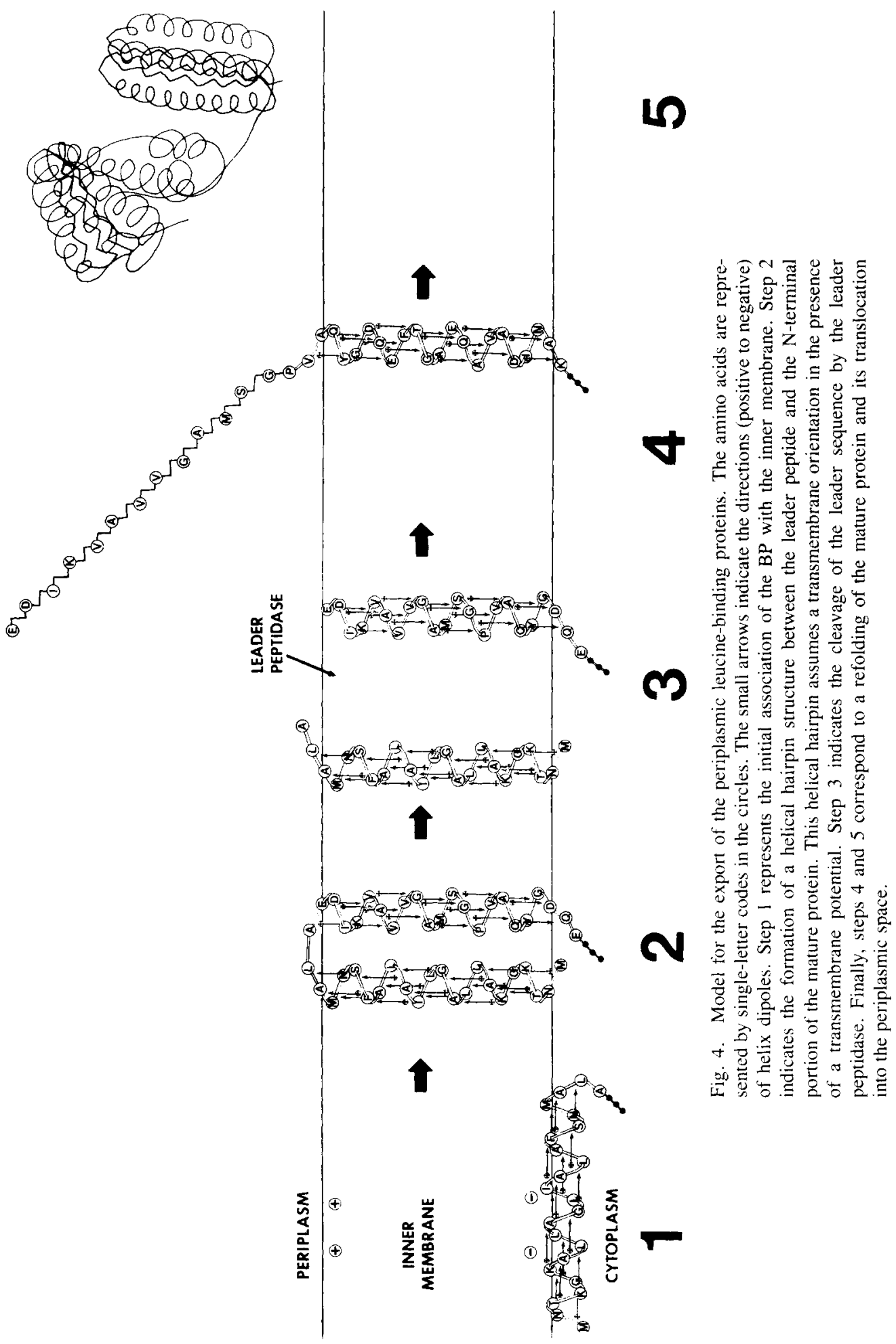


4. In this model, a major role of the membrane potential is suggested for the proper membrane orientation of the proposed helix hairpin formed by the signal sequence and the first segment of the mature portion of the BP $[16,17]$.

Recent work on the secretion of the BPs has been directed toward identifying the sequences required to achieve secretion. To this end, mutants of LS-BP carrying various deletions or sequence alterations have been constructed [17]. It was found that these altered binding proteins were processed and secreted normally. Moreover, hybrid molecules were constructed between the tryptophan synthetase alpha-subunit and various lengths of the N-terminal portion of the LS-BP [18]. The results showed that almost the entire C-terminal half of the LS-BP can be removed with no adverse effect on processing or secretion of the hybrid protein. Thus, the signal sequence together with a portion of the N-terminal portion of the LS-BP is sufficient to direct its export into the periplasmic space.

\section{THE BPS AS MODELS IN STUDYING STRUCTURE-FUNCTION RELATIONSHIPS}

Because the LIV-BP and LS-BP have three known functions in $E$ coli, they are proving useful in examining the relationships between protein structure and function. For example, using oligonucleotide-directed mutagenesis, the nonhomologous residues in the clefts of the BPs are being altered. If an alteration corresponds with a change in either substrate affinities or substrate specificities, then knowledge will be gained about the mechanism of substrate binding. Other changes are being directed toward the regions of high amino acid homology on the BPs that are potential regions for interacting with the membrane components. These altered BPs will be especially useful for study when the individual membrane proteins have been isolated. Finally, work in progress also involves analyzing additional LS-BP-trpA hybrid molecules containing larger $\mathrm{C}$-terminal deletions to identify more precisely the $\mathrm{N}$-terminal sequences required for export of the BPs into the periplasmic space. Since the LIV$\mathrm{BP}$ and LS-BP are the products of gene duplication, a comparison of their structurefunction relationships should help illuminate the potential constraints placed on their evolutionary divergence.

In conclusion, the leucine binding proteins of $E$ coli have been thoroughly studied by biochemical, genetic, and recombinant DNA approaches. As a consequence, much is already known about their molecular structure and their relationship to each other. Because these proteins must be secreted and interact with the same membrane components yet exhibit divergent substrate specificities, they provide a model system for studying the relationship of protein structure to protein function.

\section{REFERENCES}

1. Piperno JR, Oxender DL: J Biol Chem 243:5914-5920, 1968.

2. Rahmanian M. Claus DR, Oxender DL: J Bacteriol 116:1258-1266, 1973.

3. Wood JM: J Biol Chem 250:4477-4485, 1975.

4. Anderson JJ, Oxender DL: J Bacteriol 136:168-174, 1978.

5. Oxender DL, Anderson JJ, Daniels CJ, Landick R, Gunsalus RP, Zurawski G, Selker E, Yanofsky C: Proc Natl Acad Sci USA 77:1412-1416, 1980

6. Nazos PN: Ph.D. Thesis, University of Michigan, Ann Arbor, 1984.

7. Landick R: Ph.D. Thesis, University of Michigan, Ann Arbor, 1983. 
8. Landick R, Oxender DL: J Biol Chem, 260:8257-8261, 1985.

9. Landick R, Anderson JJ, Mayo MM, Gunsalus RP, Mavromara P, Daniels CJ, Oxender DL: J Supramolec Struct 14:527-537, 1981.

10. Oxender DL, Anderson JJ, Daniels CJ, Landick R, Gunsalus RP, Zurawski G, Yanofsky C: Proc Natl Acad Sci USA 77:2005-2009, 1980.

11. Riley M, Aninlionis A: Annu Rev Microbiol 32:519-560, 1978.

12. Ovchinnikov YA, Aldanova NA, Grinkevich VA, Arzamazova NM, Moroz IN, Nazimov IV: FEBS Lett 78:313-316, 1977.

13. Saper M, Quiocho FA: J Biol Chem 258:11057-11062, 1983.

14. Daniels CJ, Anderson JJ, Landick R, Oxender DL: J Supramolec Struct 14:305-311, 1980.

15. Chou TY, Fasman GD: Annu Rev Biochem 47:251-276, 1978.

16. Daniels CJ, Bole DG, Quay SC, Oxender DL: Proc Natl Acad Sci USA 78:5396-5400, 1981.

17. Landick R, Duncan JR, Copeland BR, Nazos PM, Oxender DL: J Cell Biochem 24:331-344, 1984.

18. Su TZ, Copeland BR, Landick R, Oxender DL: J Biol Chem 1985, (submitted). 